\title{
Outage Analysis of Multihop Wireless Backhaul Using Millimeter Wave under Blockage Effects
}

\author{
Haejoon Jung ${ }^{1}$ and In-Ho Lee ${ }^{2}$ \\ ${ }^{1}$ Department of Information and Telecommunication Engineering, Incheon National University, Incheon 22012, Republic of Korea \\ ${ }^{2}$ Department of Electrical, Electronic and Control Engineering, Hankyong National University, Anseong 17579, Republic of Korea \\ Correspondence should be addressed to In-Ho Lee; ihlee@hknu.ac.kr
}

Received 20 November 2016; Revised 30 January 2017; Accepted 5 February 2017; Published 21 February 2017

Academic Editor: Mihajlo Stefanovic

Copyright (c) 2017 Haejoon Jung and In-Ho Lee. This is an open access article distributed under the Creative Commons Attribution License, which permits unrestricted use, distribution, and reproduction in any medium, provided the original work is properly cited.

\begin{abstract}
We consider multihop millimeter-wave (mm-Wave) wireless backhaul communications, by which small cell base station (SBS) clusters can connect to a macrocell base station (MBS). Assuming the mm-Wave wireless backhaul links suffer from outage caused by obstacles that block the line-of-sight (LoS) paths, we derive the statistics of a perhop distance based on the blockage model using stochastic geometry and random shape theory and analyze the multihop outage probability using the statistics of a perhop distance. We also provide an optimal number of hops to minimize the end-to-end outage performance between the MBS and the destination SBS cluster when the end-to-end distance is given.
\end{abstract}

\section{Introduction}

Dense deployment of small cells over traditional macrocell s is considered as a key enabling technique for the emerging fifth-generation (5G) cellular networks [1-4]. For the small cell network deployment, millimeter-wave ( $\mathrm{mm}$-Wave) wireless backhaul is a cost-effective and scalable solution with large contiguous bandwidths. Moreover, the line-of-sight (LoS) nature of $\mathrm{mm}$-Wave communication with directional antenna arrays can help to control the signal interference [5].

However, mm-Wave signals are more sensitive to blockage effects than signals in lower frequency bands, as indicated by the measurement data in [6-8]. Thus, the performances of the mm-Wave cellular systems are vastly affected by blockage effects [9]. There are two widely used approaches to incorporate the impact of blockages into signal propagation: ray tracing and stochastic modeling. In the ray tracing approach, blockages are characterized explicitly by their sizes, locations, and shapes. Therefore, this method is appropriate for environment-specific simulations based on electromagnetic simulation tools, which perform complex numerical calculations for ray tracing $[10,11]$. On the other hand, in the stochastic models, the statistics of blockages are characterized with smaller number of parameters compared to ray tracing. Therefore, the stochastic models are used to analyze general networks with acceptable accuracy.

In [12], the authors propose mm-Wave channel model incorporating the blockage effects based on stochastic geometry and random shape theory. Stochastic geometry has been a powerful technique to evaluate system performance in the conventional cellular networks [13], which reveals the impacts of multiple system parameters such as base station density, transmit power, and path-loss exponent. The key idea in [12] is to model random obstacles (e.g., buildings) as rectangles with random sizes and orientations whose centers form a Poisson point process (PPP) on 2-dimensional space. The model proposed in [12] can capture distance-dependent characteristics of the blockage effects, which is more realistic compared to the conventional shadowing model using lognormal distribution.

Using this mm-Wave propagation model in [10], the authors in [14] present a framework to derive signal-tointerference-plus-noise ratio (SINR) distributions, which can be used to analyze coverage and rate performances. Also, the 
outage probability of a macrodiversity system with multiple base stations (BSs) that are connected by wire to each other is analyzed in [15], where an outage occurs when there is no LoS path from all the base stations to the user. The analysis in [15] presents that this macrodiversity coming from the unblocked BS selection can be exploited to mitigate blockage in $\mathrm{mm}-$ Wave cellular systems.

In [16], the outage performance of $\mathrm{mm}$-Wave wireless backhaul links between a macrocell base station (MBS) and small cell base stations (SBSs) is also studied using the channel model in [10]. Specifically, in [16], the multiple SBSs in a cluster have wired connections to each other, and the wireless backhaul link between the MBS and the SBS cluster is assumed to be reliable as long as there exists one or more SBSs that have blockage-free LoS paths from the MBS. In other words, the MBS can selectively choose an unblocked SBS to construct a wireless backhaul link by beam steering, which is equivalent to the macrodiversity in [15].

However, while [16] only considers the one-hop communication between the MBS and a single SBS cluster, the $5 \mathrm{G}$ cellular networks may have multiple SBS clusters, which require multihop transmissions to improve the cell coverage $[1,2]$. Considering the distance dependence of the blockage effects (i.e., the likelihood of a blockage event increases as the distance increases) at mm-Wave, multihop communication can be an effective solution to build mm-Wave wireless backhaul systems. In this context, motivated by the limitation in [16], we extend the single-hop wireless backhaul system in [16] to a multihop scenario with multiple SBS clusters. Moreover, it is noteworthy that the wireless backhaul links between two SBS clusters studied in this paper are distinct from the system model in [16] because we consider multiplepoints-to-multiple-points (SBSs-to-SBSs) links, while [16] is focused on single-point-to-multiple-points (MBS-to-SBSs) links. Therefore, with different distance statistics from [16], the intercluster SBS-to-SBS communication can benefit from higher order of spatial diversity compared with the MBS-toSBS communication in [16].

The contributions of this paper are fourfold. First, we derive the probability distribution, mean, and variance of a perhop distance (i.e., the distance between two randomly located SBSs in two adjacent SBS clusters), considering the spatial diversity. Second, we analyze the perhop outage performance of mm-Wave wireless backhaul links between two adjacent SBS clusters. Third, the outage analysis is extended from the perhop link to multihop systems. Lastly, we present an optimal and suboptimal hop count to minimize the end-to-end outage performance between the MBS and the destination SBS cluster for a given end-to-end distance, where the suboptimal hop count is derived based on only the perhop outage performance.

This paper is organized as follows. We introduce the system model in Section 2. We derive the probability distribution, mean, and variance of the distance between two SBS clusters in Section 3. The outage performances of perhop and multihop cases are analyzed in Section 4. In Section 5, we show optimal hop distance and hop count to minimize the outage performance and propose suboptimal hop distance and hop count that give close enough outage probabilities

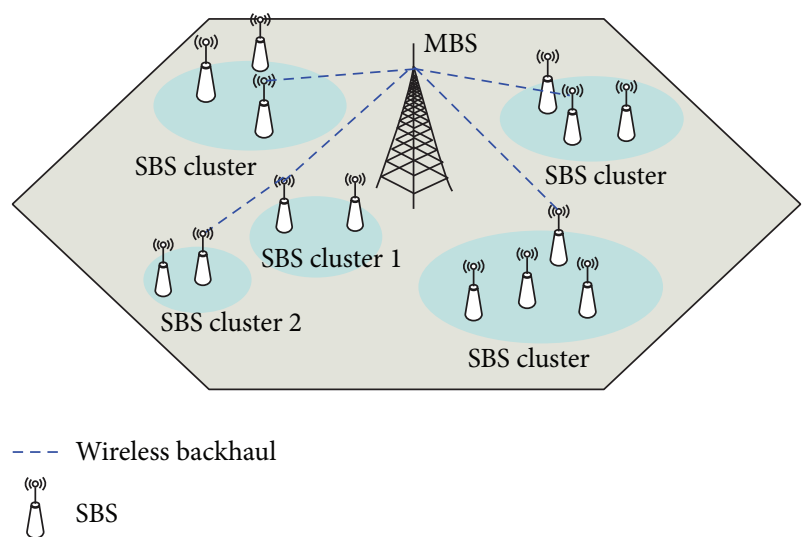

FIGURE 1: Illustration of a 5G-network with mm-Wave wireless backhaul.

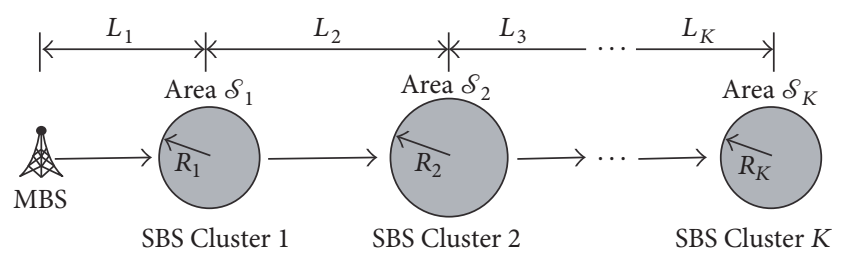

FIGURE 2: Multihop wireless backhaul.

to the optimal ones. Finally, conclusions are provided in Section 6.

\section{System Model}

We consider a 5G network with a single MBS and multiple SBS clusters as an example illustration in Figure 1. In the figure, the macrocell is indicated by the gray hexagon, and the SBS clusters are represented by the sky blue circles. Each SBS cluster has multiple SBSs, which are connected to each other through wires to enable spatial diversity in SBS cluster $[15,16]$. The MBS is connected to the core network, and SBSs can access the core network via the MBS using wireless backhaul, which is indicated by the dotted blue lines in the figure. Moreover, some SBS clusters, which are far from the MBS, trigger multihop transmissions for the backhaul links to overcome path loss and blockage effects. For example, in Figure 1, SBS Cluster 2 communicates with the MBS in two hops over Cluster 1.

As shown In Figure 2 focusing on the multihop wireless backhaul links, we assume that SBSs in each cluster are distributed over a circle. In the figure, the areas of the multihop clusters are denoted by Areas $\delta_{1}, \delta_{2}, \ldots$, and $\delta_{K}$ with radii of $R_{1}, R_{2}, \ldots, R_{K}$, respectively. Also, the hop distances are denoted by $L_{k}$, where $k \in\{1,2, \ldots, K\}$. As in [16], we assume that in $k$-th cluster multiple SBSs are uniformly distributed with intensity $\lambda_{k}$ according to a homogeneous point process (PPP) [17], which is a widely used model for various types of 


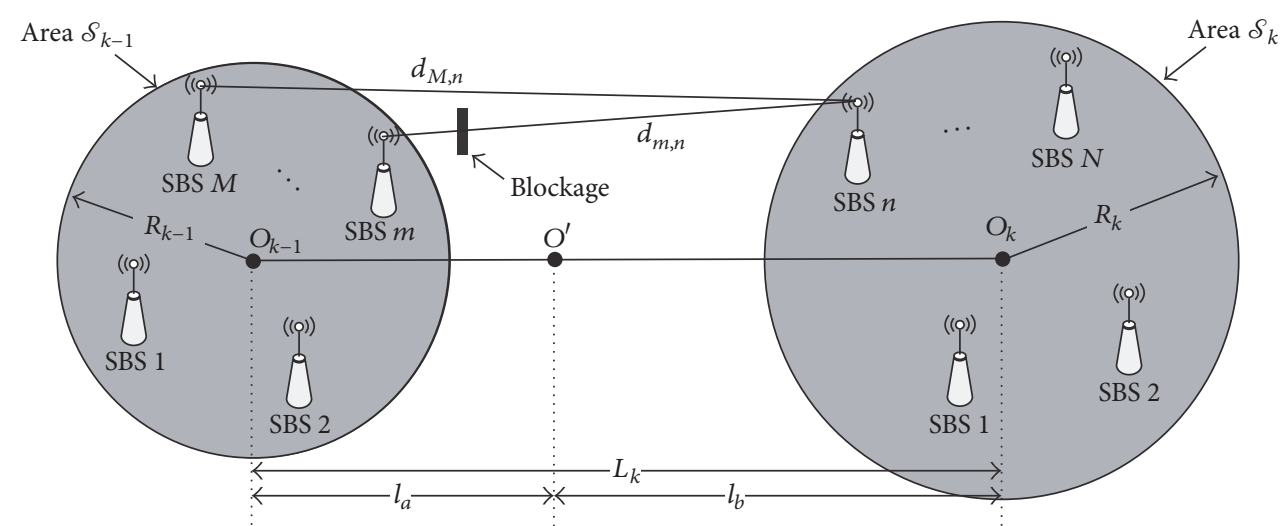

FIGURE 3: Intercluster mm-Wave wireless backhaul links.

cellular networks [18]. For the end-to-end wireless backhaul link with hops, the outage probability can be expressed as

$$
P_{\text {out }}=1-\prod_{k=1}^{K}\left(1-P_{\text {out }: k}\right),
$$

where $P_{\text {out: } k}$ is the outage probability of $k$-th hop [19]. Assuming the outage events in different hops are mutually independent, the outage analysis over multiple hops can be decoupled into multiple intercluster links, while the firsthop outage $P_{\text {out: } 1}$ can be obtained based on the analysis in [16]. When we consider the multihop performance $P_{\text {out }}$, we assume uniform SBS clusters and equidistant hops for the mathematical tractability which we will explain in detail in Section 4.2. In general, however, the densities of the SBS clusters are dissimilar, which means variable $P_{\text {out: } k}$ and hop distances for different $k$. Even in this case, the perhop analysis in this paper still can be used to optimize the end-to-end outage $P_{\text {out }}$, which is a function of the hop distances and the corresponding end-to-end hop count $K$.

To analyze the outage performances of the following hops, we consider the intercluster outage $P_{\text {out: } k}$ for $k \geq 2$ as shown in Figure 3. Clusters $k-1$ and $k$ have $M$ and $N$ SBSs, which are uniformly distributed over $\mathcal{S}_{k-1}$ and $\mathcal{S}_{k-2}$ with intensities $\lambda_{k-1}$ and $\lambda_{k}$, respectively. These intensities correspond to the average numbers of SBSs per unit area (i.e, SBSs $/ \mathrm{m}^{2}$ ). In other words, the numbers of the SBSs in the two clusters are assumed to be $M$ and $N$, which are independent random variables following Poisson probability distributions as

$$
\begin{aligned}
& \operatorname{Pr}[M=m]=\frac{\left(\lambda_{k-1} \pi R_{k-1}^{2}\right)^{m}}{m !} e^{-\lambda_{k-1} \pi R_{k-1}^{2},} \\
& \operatorname{Pr}[N=n]=\frac{\left(\lambda_{k} \pi R_{k}^{2}\right)^{n}}{n !} e^{-\lambda_{k} \pi R_{k}^{2}},
\end{aligned}
$$

where $\operatorname{Pr}[X=x]$ denotes the probability that $X=x$ and $x$ is a nonnegative integer. Therefore, the average numbers of the SBSs in the two clusters are given by $\mathbb{E}\{M\}=\lambda_{k-1} \pi R_{k-1}^{2}$ and $\mathbb{E}\{N\}=\lambda_{k} \pi R_{k}^{2}$, where $\mathbb{E}\{\cdot\}$ implies the expectation operator. We assume the centers of the two clusters are separated by $L_{k}$ with fixed ratios to the two radii as $L_{k}=a_{k} R_{k-1}=b_{k} R_{k}$, where $a_{k}>2$ and $b_{k}>2$ (i.e., there is no intersection between $\mathcal{S}_{k-1}$ and $\mathcal{S}_{k}$ ) (we assume the dense deployment of SBSs with wired connections in each cluster for coverage extension, especially to solve the hotspots problem, and the geographically disjoint SBS clusters. In addition, considering the construction cost, we assume that the SBSs in different clusters communicate with each other through the multihop wireless backhaul.).

Let $d_{m, m}$ be the distance between the mm-Wave SBSs $m$ and $n$ in $\mathcal{S}_{k-1}$ and $\mathcal{S}_{k}$, respectively, as shown in the figure. Also, as in $[12,14-16]$, the probability of no blockage in an individual SBS-to-SBS (S2S) path is $P_{\operatorname{LoS}(m, n)}=e^{-\beta d_{m, n}}$, where $\beta$ is the parameter that captures density and size of obstacles. The greater $\beta$ means obstacles with higher density and larger sizes, which results in lower $P_{\operatorname{LoS}(m, n)}$. In this paper, we assume $P_{\operatorname{LoS}(m, n)}$ can be interpreted as the probability that the communication link between the SBSs $m$ and $n$ in the two clusters is reliable.

As in [16], we assume that the multiple SBSs in the same cluster are connected to each other through wires and thus the wireless backhaul link between the two clusters is reliable if one (or more) of the individual S2S links between the two clusters is reliable. An outage event of the intercluster mmWave wireless backhaul link is caused by blockage of LoS path. Before data transmission, with beam steering, each SBS in Cluster $k-1$ can selectively connect to one of the SBSs in Cluster $k$ whose LoS path is blockage-free. Also, once one SBS finds an unobstructed LoS path, the SBS notifies the other SBSs in the same cluster via the wired intracluster connections.

\section{Probability Distribution of S2S Distance $d_{m, n}$ between Two SBS Clusters}

In order to investigate the performance of the mm-Wave wireless backhaul system, we need to consider probability distribution of the S2S distance $d_{m, n}$ between the two SBS clusters, since $P_{\operatorname{LoS}(m, n)}$ is a function of $d_{m, n}$, which is a random variable. As shown in Figure 4(a), suppose the distance between the center of the left SBS cluster $O_{k-1}$ and SBS $n$ in the right SBS cluster is $v_{n}$. To derive the probability distribution of 


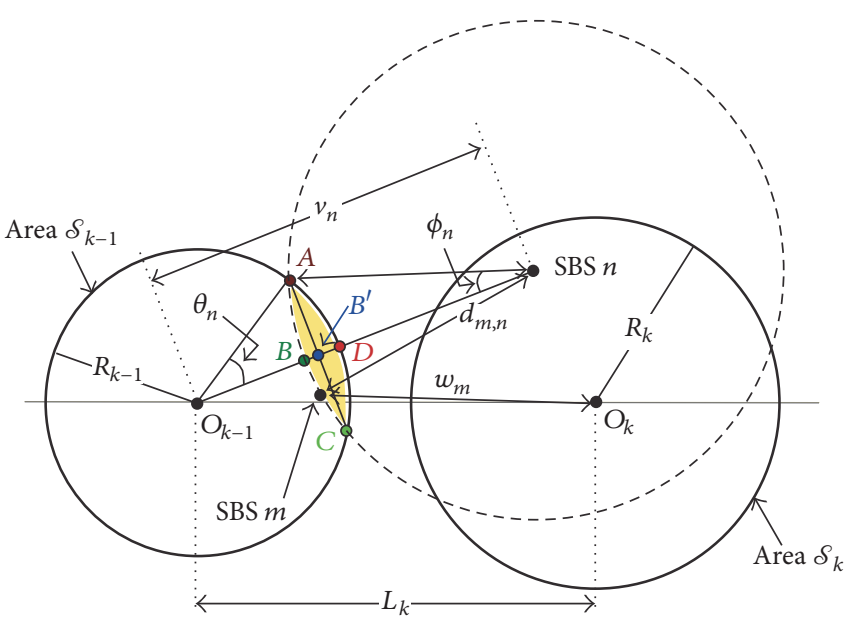

(a)

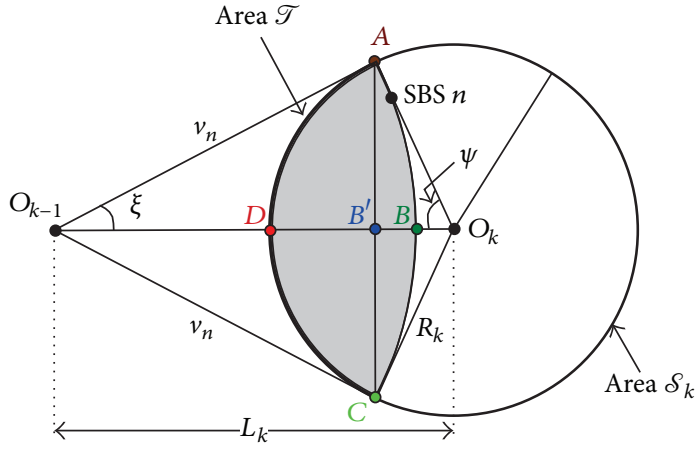

(b)

Figure 4: An example illustration to derive the PDF of $d_{m, n}$.

$d_{m, n}$, we first find the probability distribution function (PDF) of $v_{n}$ using an example illustration in Figure 4 (because $v_{n}$ is the distance between a random location in Area $\delta_{k}$ and the fixed point $O_{k-1}$, the PDF of $v_{n}$ is identical to the PDF of the distance of the single-hop wireless backhaul in [16].), and then conditioned on $v_{n}$ we will obtain the PDF of $d_{m, n}$. In Figure 4(b), the shaded area (Area $\mathscr{T}$ ) is the feasible region of $v_{n}$ in the circle (Area $\mathcal{S}_{k}$ ). Consequently, the cumulative density function (CDF) of $v_{n}$ is expressed as the ratio between Areas $\mathscr{T}$ and $\delta_{k}$ as

$$
F_{v_{n}}(x)=\operatorname{Pr}\left[v_{n} \leq x\right]=\frac{\psi R_{k}^{2}+\xi x^{2}-R_{k} L_{k} \sin \psi}{\pi R_{k}^{2}},
$$

Where $L_{k}-R_{k} \leq x \leq L_{k}+R_{k}, \psi=\arccos \left(\left(R_{k}^{2}+L_{k}^{2}-\right.\right.$ $\left.\left.x^{2}\right) / 2 R_{k} L_{k}\right)$, and $\xi=\arccos \left(\left(x^{2}+L_{k}^{2}-R_{k}^{2}\right) / 2 x L_{k}\right)$. As a result, the PDF of $v_{n}$ is

$$
\begin{gathered}
f_{v_{n}}(x)=\frac{x}{\pi R_{k}^{2}}\left(\frac{R_{k} / L_{k}}{\sqrt{1-\left(L_{k}^{2}-x^{2}+R_{k}^{2}\right)^{2} / 4 L_{k}^{2} R_{k}^{2}}}\right. \\
-\frac{\left(x / L_{k}-\left(L_{k}^{2}+x^{2}-R_{k}^{2}\right) / 2 L_{k} x\right)}{\sqrt{1-\left(L_{k}^{2}+x^{2}-R_{k}^{2}\right)^{2} / 4 L_{k}^{2} x^{2}}} \\
-\frac{\left(L_{k}^{2}-x^{2}+R_{k}^{2}\right) / 2 L_{k} R_{k}}{\sqrt{1-\left(L_{k}^{2}-x^{2}+R_{k}^{2}\right)^{2} / 4 L_{k}^{2} R_{k}^{2}}} \\
\left.+2 \arccos \left(\frac{L_{k}^{2}+x^{2}-L_{k}^{2}}{2 L_{k} x}\right)\right),
\end{gathered}
$$

where $L_{k}-R_{k} \leq x \leq L_{k}+R_{k}$. As shown in [16], when $L_{k}>$ $2 R_{k}$, we can derive an approximate PDF by treating the area enclosed by the path $\overline{A B C D A}$ as the region bounded by the path $\overline{A B^{\prime} C D A}$ in Figure 4(b):

$$
f_{v_{n}}^{*}(x)=\frac{2 \sqrt{R_{k}^{2}-\left(L_{k}-x\right)^{2}}}{\pi R_{k}^{2}},
$$

where $L_{k}-R_{k} \leq x \leq L_{k}+R_{k}$. With this approximated PDF, we can obtain $\mathbb{E}\left\{v_{n}\right\}=L_{k}$ and $\mathbb{V A R}\left\{v_{n}\right\}=R_{k}^{2} / 4$.

Then, going back to Figure 4(a), conditioned on $v_{n}$, the PDF of $d_{m, n}$ can be derived as the ratio of the area within a closed path $\overline{A B C D A}$ to the entire circle $S_{k-1}$, which is given by

$$
F_{d_{m, n} \mid v_{n}}\left(x \mid v_{n}\right)=\frac{\theta_{n} R_{k-1}^{2}+\phi_{n} x^{2}-R_{k-1} v_{n} \sin \theta_{n}}{\pi R_{k-1}^{2}},
$$

where $v_{n}-R_{k-1} \leq x \leq v_{n}+R_{k-1}, \theta_{n}=\arccos \left(\left(R_{k-1}^{2}+L_{k}^{2}-\right.\right.$ $\left.\left.x^{2}\right) / 2 R_{k-1} v_{n}\right)$, and $\phi_{n}=\arccos \left(\left(x^{2}+v_{n}^{2}-R_{k-1}^{2}\right) / 2 x v_{n}\right)$. Hence, the corresponding conditional PDF of $d_{m, n}$ is given by

$$
\begin{aligned}
& f_{d_{m, n} \mid v_{n}}\left(x \mid v_{n}\right)=\frac{d F_{d_{m, n} \mid v_{n}}\left(x \mid v_{n}\right)}{d x} \\
& =\frac{x}{\pi R_{k-1}^{2}}\left(\frac{R_{k-1} / v_{n}-\left(v_{n}^{2}-x^{2}+R_{k-1}^{2}\right) / 2 v_{n} R_{k-1}}{\sqrt{1-\left(v_{n}^{2}-x^{2}+R_{k-1}^{2}\right)^{2} / 4 v_{n}^{2} R_{k-1}^{2}}}\right. \\
& -\frac{x / v_{n}-\left(v_{n}^{2}+x^{2}-R_{k-1}^{2}\right) / 2 v_{n} x}{\sqrt{1-\left(v_{n}^{2}+x^{2}-R_{k-1}^{2}\right)^{2} / 4 v_{n}^{2} x^{2}}} \\
& \left.+2 \arccos \left(\frac{v_{n}^{2}+x^{2}-R_{k-1}^{2}}{2 v_{n} x}\right)\right) .
\end{aligned}
$$


As the PDF of $v_{n}$, for a large enough $L_{k}$ relative to $R_{k}$ and $R_{k-1}$, this conditional PDF has an approximate form

$$
f_{d_{m, n} \mid v_{n}}^{*}\left(x \mid v_{n}\right)=\frac{2 \sqrt{R_{k-1}^{2}-\left(v_{n}-x\right)^{2}}}{\pi R_{k-1}^{2}},
$$

where $v_{n}-R_{k-1} \leq x \leq v_{n}+R_{k-1}$. Hence, the PDF of $d_{m, n}$ is given by

$$
\begin{gathered}
f_{d_{m, n}}^{*}(x)=\int_{L-R_{k}}^{L+R_{k}} f_{d_{m, n} \mid v_{n}}^{*}\left(x \mid v_{n}=y\right) f_{v_{n}}^{*}(y) d y \\
=\int_{L-R_{k}}^{L+R_{k}} \frac{2 \sqrt{R_{k-1}^{2}-(y-x)^{2}}}{\pi R_{k-1}^{2}} \frac{2 \sqrt{R_{k}^{2}-(L-y)^{2}}}{\pi R_{k}^{2}} d y,
\end{gathered}
$$

where $L_{k}-R_{k-1}-R_{k} \leq x \leq L_{k}+R_{k-1}+R_{k}$. This approximate $\operatorname{PDF} f_{d_{m, n}}^{*}(x)$ gives the mean and variance as follows:

$$
\begin{aligned}
\mathbb{E}\left\{d_{m, n}\right\}= & \mathbb{E}\left\{\mathbb{E}\left\{d_{m, n} \mid v_{n}\right\}\right\}=\mathbb{E}\left\{v_{n}\right\}=L_{k}, \\
\mathbb{V A \mathbb { R }}\left\{d_{m, n}\right\}= & \mathbb{E}\left\{\mathbb{V A R}\left\{d_{m, n} \mid v_{n}\right\}\right\} \\
& +\mathbb{V} \mathbb{R}\left\{\mathbb{E}\left\{d_{m, n} \mid v_{n}\right\}\right\} \\
= & \frac{R_{k-1}^{2}}{4}+\mathbb{V A \mathbb { R }}\left\{v_{n}\right\}=\frac{R_{k-1}^{2}+R_{k}^{2}}{4} .
\end{aligned}
$$

These two statistics will be used to derive the perhop outage probability in the next section.

\section{Outage Analysis}

4.1. Intercluster Outage Analysis. In this section, we derive the probability that there is no reliable S2S LoS link between the two SBS clusters $\mathcal{S}_{k-1}$ and $\mathcal{S}_{k}$. We define an outage, if all the LoS S2S paths are blocked by obstacles. Let $U_{m, n}$ be a Bernoulli random variable as

$$
U_{m, n}= \begin{cases}1, & \text { w.p. } P_{\operatorname{LoS}(m, n)}=e^{-\beta d_{m, n}}, \\ 0, & \text { w.p. } 1-P_{\operatorname{LoS}(m, n)}=1-e^{-\beta d_{m, n}},\end{cases}
$$

where if $U_{m, n}=1$, it means that the mm-Wave link between SBS $m$ in $\mathcal{S}_{k-1}$ and SBS $n$ in $\mathcal{S}_{k}$ is reliable. Assuming that blockage is impenetrable, as in [12, 14-16], an outage event of the mm-Wave SBS occurs, if all $U_{m, n}$ 's are zeros. In other words, if there is at least one $\operatorname{LoS}$ mm-Wave link with $U_{m, n}=1$ for any pair $m$ and $n$, the $\mathrm{mm}$-Wave communication between the two SBS clusters is reliable. Therefore, the outage in $k$ th hop can be defined as

$$
\begin{aligned}
& P_{\text {out: } k} \\
& =\sum_{m=1}^{\infty} \sum_{n=1}^{\infty} \operatorname{Pr}\left[U_{i, j} \text { 's are all zeros } \mid M=m, N=n\right] \\
& \text { - } \operatorname{Pr}[M=m, N=n]+\operatorname{Pr}[M=0] \\
& +\operatorname{Pr}[N=0] \text {, }
\end{aligned}
$$

where $1 \leq i \leq m$ and $1 \leq j \leq n$.
Following $[12,14-16]$, for analytical tractability, we assume the outage events indicated by $U_{i, j}$ 's in (12) are mutually independent for all $i$ and $j$. Strictly speaking, the blockage events on different links are not always independent. Therefore, as in $[15,16]$, the outage probability in this paper may be a lower bound as a reference of system design and analysis. However, we note that the numerical results in [12] show that the error caused by the independent link assumption is minor and acceptable in accuracy. With the assumption of the independent blockage events, we have

$$
\begin{aligned}
\operatorname{Pr} & {\left[U_{i, j} \text { 's are all zeros } \mid M=m, N=n\right] } \\
& =\prod_{j=1}^{n} \prod_{i=1}^{m} \mathbb{E}\left[1-e^{-\beta d_{i j}}\right]=\left(E\left[1-e^{-\beta d_{i j}}\right]\right)^{m n},
\end{aligned}
$$

where the distance $d_{i j}$ between SBSs $i$ and $j$ from $\mathcal{S}_{k-1}$ and $\mathcal{S}_{k}$ is a random variable with the approximate PDF in (9). Letting $A=\mathbb{E}\left\{1-e^{-\beta d_{i j}}\right\}$, it can be approximated by Taylor expansion as

$$
\begin{aligned}
A & \approx 1-\int_{L-R_{k-1}-R_{k}}^{L+R_{k-1}+R_{k}} e^{-\beta x} f_{d_{i j}}^{*}(x) d x \\
& \approx 1-\left(e^{-\beta \mathbb{E}\left\{d_{i, j}\right\}}+\frac{\beta^{2}}{2} e^{-\beta \mathbb{E}\left\{d_{i, j}\right\}} \mathbb{V A \mathbb { R }}\left\{d_{i, j}\right\}\right) \\
& \approx 1-\left(1+\frac{\beta^{2}\left(R_{k-1}^{2}+R_{k}^{2}\right)}{8}\right) e^{-\beta L_{k}},
\end{aligned}
$$

where $L=a_{k} R_{k-1}=b_{k} R_{k}$. Thus, the outage probability in (12) is given by

$$
P_{\text {out }: k}=\sum_{m=0}^{\infty} \sum_{n=0}^{\infty} A^{m n} \operatorname{Pr}[N=n] \operatorname{Pr}[M=m] .
$$

Thus, we can interpret $A$ in (14) as the expected $P_{\text {out: } k}$, when the number of SBSs in the two clusters is given $(N=n$ and $M=m$ ). Unfortunately, it is difficult to obtain a closed form expression of (15), but based on (14) and (15), we can observe some important properties of the intercluster outage probability $P_{\text {out: } k}$ as follows.

Property 1. For given positive $\beta, \lambda_{k-1}, \lambda_{k}, a_{k}$, and $b_{k}, P_{\text {out: } k} \rightarrow$ 1 as $L_{k} \rightarrow 0$ (because $R_{k-1}=L_{k} / a_{k} \rightarrow 0$ and $R_{k}=L_{k} / b_{k} \rightarrow$ 0 ) (as described in Section 2, we assume the two SBS clusters are disjoint by $L_{k}=a_{k} R_{k-1}=b_{k} R_{k}$ with $a_{k}>2$ and $b_{k}>2$. Thus, when $L_{k} \rightarrow 0$, the sizes of the two clusters also become zero, which triggers an outage since the clusters do not have any SBSs.). Moreover, as $L_{k} \rightarrow \infty, P_{\text {out: } k} \rightarrow 1$. This property can be proved by $A \rightarrow 1$, as $L_{k} \rightarrow 0$ or $\infty$.

Property 2. For fixed $\beta, \lambda_{k-1}, \lambda_{k}, R_{k-1}$, and $R_{k}$, as $L_{k}$ increases (i.e., $a_{k}$ and $b_{k}$ increase), $P_{\text {out }: k}$ increases, because $A$ increases.

Property 3. When the other parameters are fixed, the greater $\beta$ gives the higher $P_{\text {out: } k}$.

Property 4. As $\lambda_{k-1}$ and $\lambda_{k}$ increase, $P_{\text {out: } k}$ decreases because the expected numbers of the SBSs $M$ and $N$ increase. 


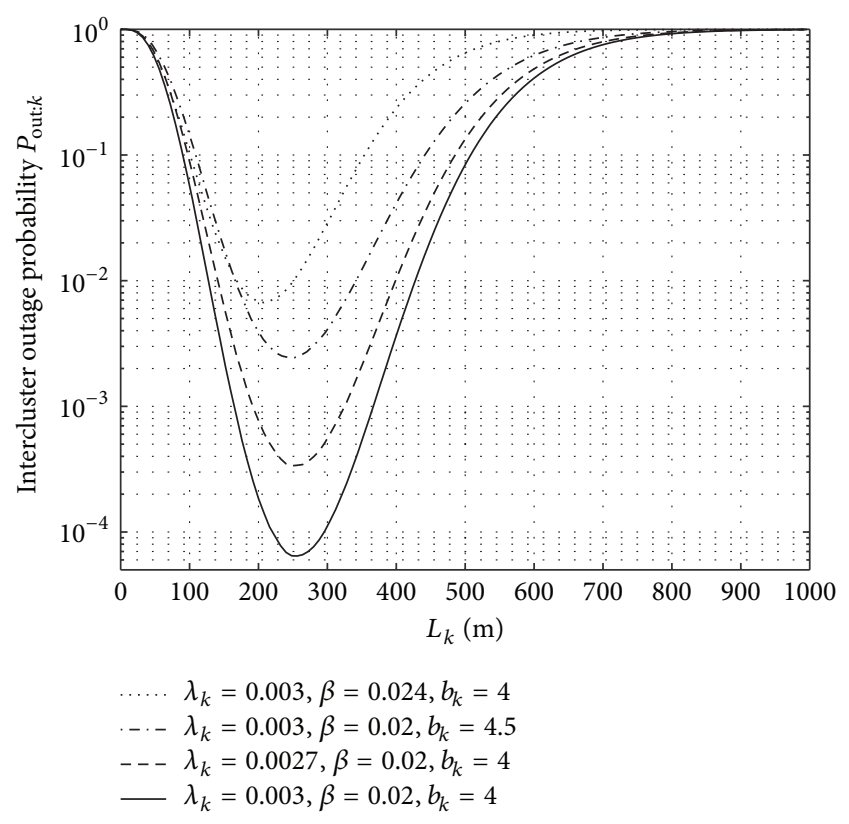

FIGURE 5: Intercluster outage probability $P_{\text {out: }}$ versus $L_{k}$.

Figure 5 displays simulation results of the intercluster outage, where the horizontal axis indicates the hop distance $L_{k}$, while the vertical axis represents the intercluster outage probability $P_{\text {out }: k}$. Assuming $R_{k}=R_{k-1}, \lambda_{k}=\lambda_{k-1}$, and $a_{k}=b_{k}$, the solid line is the baseline, while the other three graphs correspond to changes in $\beta, \lambda_{k}$, and $b_{k}$, respectively. We can observe all the four properties in Figure 5 . To be specific, all the four graphs in the figure are convex functions of $L$, which corresponds to Property 1 . Also, as described in Property 2, compared to the solid line (i.e., the baseline case), the dash-dot graph, which indicates the higher $a_{k}=b_{k}$, shows the higher outage rates. Property 3 can be observed by the comparison between the solid and the dotted curves. Lastly, Property 4 is found by the dashed line in the figure.

\subsection{Multihop Outage Analysis with a Constant Hop Distance.} In this section, we extend the outage analysis to the multihop wireless backhaul assuming the uniform SBS clusters (i.e., $L_{k}=L, R_{k}=R$, and $\lambda_{k}=\lambda$ for all $k$ ). With this assumption, the end-to-end outage probability in (1) between the MBS and the $K$-th SBS cluster is simplified as

$$
P_{\text {out }}=1-\left(1-P_{\text {out: } 1}\right)\left(1-P_{\text {out: } k}\right)^{K-1},
$$

where $P_{\text {out: } 1}$ is the outage probability for the first-hop link between the MBS and the first SBS cluster $\mathcal{S}_{1}$ presented in [16] and $P_{\text {out: } k}$ is given in (15). Because the height of the MBS is typically much greater than that of SBS, based on [12], we need to use a smaller blockage parameter $\beta^{\prime}=c \beta$ for the first hop, where $c<1$. Thus, $P_{\text {out: } 1}<P_{\text {out: } k \text {. When the end-to- }}$ end distance between the MBS to the center of the $K$-th SBS cluster $O_{K}$ is $D$, then the hop distance is $L=D / K$.

Figure 6 shows multihop simulation results with $c=1 / 4$, where the $x$-and $y$-axes are the number of hops $K$ and the

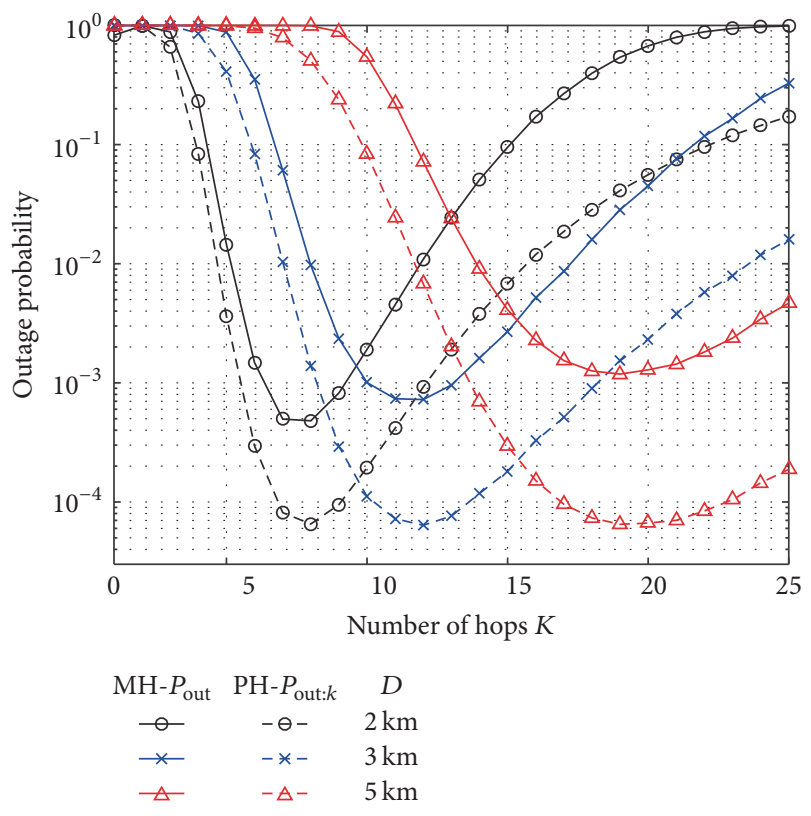

Figure 6: $P_{\text {out }}$ versus $K\left(\beta=0.02, \beta^{\prime}=\beta / 4, \lambda=0.003\right.$, and $b_{k}=$ $4)$.

outage probability, respectively. The three different curves with the different markers (circle, $x$-marker, and triangle) represent the outage probability results for $D=2,3$, and $5 \mathrm{~km}$, respectively. Also, the solid and dotted lines indicate the outage probabilities for the multihop $(\mathrm{MH})$ and the perhop $(\mathrm{PH})$ cases in (16) and (15), respectively. First, we can observe there exists an optimal hop count, denoted as $K_{\text {opt }}$, minimizes the outage probability for a given $D$. By the exhaustive search, we obtain $K_{\text {opt }}=8,12$, and 19 , for $D=2,3$, and $5 \mathrm{~km}$, which means that the corresponding optimal hop distances $L_{\text {opt }}=D / K_{\text {opt }}$ are almost the same for the three values of $D$. Also, the outage curves for both the $\mathrm{MH}$ and $\mathrm{PH}$ have the same $K_{\text {opt }}$ because of (i) almost negligible first-hop error (because $\beta^{\prime}=\beta / 4$ ) and (ii) the same convex curves of $P_{\text {out: } k}$ for $k \geq 2$ as in Figure 6 . Based on these observations, in the next section we propose the suboptimal hop count $\widehat{K}_{\text {opt }}$ by mathematically deriving the suboptimal hop distance $\widehat{L}_{\text {opt }}$, instead of the exhaustive search of $K$.

\section{Suboptimal Hop Count $\widehat{K}_{\text {opt }}$}

As shown in Figure 5, there is an optimal hop distance $L_{k}$ between two SBS clusters to minimize $P_{\text {out: } k}$. Assuming $a_{k}$ and $b_{k}$ are fixed, we derive the suboptimal $L_{k}$ by treating the intercluster S2S path as two-hop link between the two SBS clusters. For example, in Figure 3, suppose that we place an imaginary relay at $O^{\prime}$ on the straight line connecting $O_{k-1}$ and $\mathrm{O}_{k}$, assuming that the two clusters communicate with each other only through the relay. Suppose that the outage rates "from the left cluster to the relay" and "from the relay to the right cluster" are $P_{\text {out: } a}^{\prime}$ and $P_{\text {out: } b}^{\prime}$ with the distances $l_{a}$ and $l_{b}$, respectively. Then, as finding the center of mass, the best location of the relay $O^{\prime}$ corresponds to $P_{\text {out: } a}^{\prime}=P_{\text {out: } b}^{\prime}$. 
Using the outage probability between SBS clusters to the MBS in [16], $P_{\text {out: } a}^{\prime}$ and $P_{\text {out: } b}^{\prime}$ are expressed as

$$
P_{\text {out }: a}^{\prime}=\exp \left(-\lambda_{k-1} \pi R_{k-1}^{2}\left(1+\frac{\beta^{2} R_{k-1}^{2}}{8}\right) e^{-\beta l_{a}}\right)
$$

$$
\begin{aligned}
l_{a} & =\frac{L_{k}}{2}+\frac{1}{2 \beta} \ln \left[\frac{\lambda_{k-1} R_{k-1}^{2}\left(1+\beta R_{k-1}^{2}\right)}{\lambda_{k} R_{k}^{2}\left(1+\beta R_{k}^{2}\right)}\right] \\
l_{b} & =\frac{L_{k}}{2}+\frac{1}{2 \beta} \ln \left[\frac{\lambda_{k} R_{k}^{2}\left(1+\beta R_{k}^{2}\right)}{\lambda_{k-1} R_{k-1}^{2}\left(1+\beta R_{k-1}^{2}\right)}\right] \\
\widehat{L}_{\mathrm{opt}} & =\frac{-6 \sqrt[3]{4} b_{k}^{2}+8 \sqrt[3]{9 b_{k}^{2}+3 \sqrt{6 b_{k}^{4}-39 b_{k}^{2}+192 b_{k}}+32+2 \sqrt[3]{2}\left(9 b_{k}^{2}+3 \sqrt{6 b_{k}^{4}}-39 b_{k}^{2}+192 b_{k}+32\right)^{2 / 3}+16 \sqrt[3]{4}}}{3 \beta \sqrt[3]{9 b_{k}^{2}+3 \sqrt{6 b_{k}^{4}-39 b_{k}^{2}+192 b_{k}}+32}},
\end{aligned}
$$

where $R_{k-1}=L_{k} / a_{k}$ and $R_{k}=L_{k} / b_{k}$. If plugging $l_{a}$ and $l_{b}$ back into (17), the overall outage rate of the two-hop link with the imaginary relay is $P_{\text {out: } k}^{\prime}=1-\left(1-P_{\text {out: } a}^{\prime}\right)\left(1-P_{\text {out: } b}^{\prime}\right)$. The proposed suboptimal hop distance $\widehat{L}_{\text {opt }}$ is $L_{k}$ that minimizes $P_{\text {out } k}^{\prime}$. In other words, $\widehat{L}_{\text {opt }}$ corresponds to the solution of $\partial P_{\text {out: } k}^{\prime} / \partial L_{k}=0$, while $\partial^{2} P_{\text {out: } k}^{\prime} / \partial L_{k}^{2}>0$. This suboptimal $\widehat{L}_{\text {opt }}$ can be readily obtained by numerical calculation for given $a_{k}$, $b_{k}$, and $\beta$. If we assume the same SBS cluster sizes $R_{k}=R_{k-1}$ (i.e., $a_{k}=b_{k}$ ), we can obtain the suboptimal $\widehat{L}_{\text {opt }}$ in (20). Therefore, if the hop distances are the same with the uniform PPP intensities (i.e., $\lambda_{k}=\lambda$ for all $k$ ), the suboptimal hop count to reach the distance $D$ is $\widehat{K}_{\text {opt }}=\left\|D / \widehat{L}_{\text {opt }}\right\|$, where $\|x\|$ means the nearest integer to $x$.

Figures 7(a) and 7(b) show the simulation results of $L_{\text {opt }}$ and $K_{\text {opt }}$, respectively. In Figure $7(\mathrm{a})$, the horizontal axis is the blockage factor $\beta$, while the vertical axis is the optimal and suboptimal hop distances $L_{\text {opt }}$ and $\widehat{L}_{\text {opt }}$. As shown in the figure, both $L_{\text {opt }}$ and $\widehat{L}_{\text {opt }}$, which are indicated by the solid and dotted lines, decrease, as $\beta$ increases, because of the greater blockage effects. Also, comparing the two graphs, $\widehat{L}_{\text {opt }}<L_{\text {opt }}$ with the small gap in the entire range of $\beta$, which validates the proposed suboptimal hop distance. In Figure $7(\mathrm{~b})$, the $x$-axis represents the distance $D$ from the MBS to the $K$-th SBS cluster (i.e., the destination SBS cluster), while the $y$-axis indicates the optimal and suboptimal hop counts $K_{\text {opt }}$ and $\widehat{K}_{\text {opt }}$, which are denoted by the solid and dotted curves. In the figure, we can observe that $K_{\text {opt }}$ and $\widehat{K}$ are almost linearly increasing, as $D$ increases. Also, with the small difference, we observe that $\widehat{K}_{\text {opt }}>K_{\text {opt }}$ because $\widehat{L}_{\text {opt }}<L_{\text {opt }}$. Thus, based on $\widehat{K}_{\text {opt }}=K_{\text {opt }}+\epsilon$ with a small $\epsilon$, the suboptimal $\widehat{K}_{\text {opt }}$ can be used to reduce the exhaustive search range of $\widehat{K}_{\text {opt }}$.

Figure 8 shows the end-to-end outage probabilities versus $\beta$, where the solid and dotted lines correspond to the outage probabilities with $K_{\text {opt }}$ and $\widehat{K}_{\text {opt }}$, respectively. The markers represent different $\lambda$ and $D$. As shown in the figure, the gap between the outage probabilities with $K_{\text {opt }}$ and $\widehat{K}_{\text {opt }}$ becomes smaller as $\lambda$ and $D$ decrease. Especially, when $D=1 \mathrm{~km}$, the outage performance with $\widehat{K}_{\text {opt }}$ becomes extremely close to that with $K_{\text {opt }}$ as $\beta$ increases, which can be expected from the simulation results in Figure 7(b).

\section{Conclusion}

In this paper, we study the outage performance of the multihop wireless backhaul systems at mm-Wave in the presence of the blockage effects. Assuming that multiple SBSs are distributed in a cluster with the wired connection to each other and considering the spatial diversity in a cluster, the outage probability between two SBS clusters is derived. Through analysis and simulation, it is shown that there exists an optimal hop distance that minimizes the intercluster (perhop) outage probability. Then, assuming uniform SBS clusters we extend the perhop outage analysis to the multihop scenario, where we find that there exist optimal hop distance and hop count that minimize the end-to-end outage probability between the MBS and the destination SBS cluster when the end-to-end distance is given. Using the same trend of the perhop and multihop outage performances with respect to the perhop distance, we propose the suboptimal hop distance and corresponding suboptimal hop count. The suboptimal hop count shows a small gap to the optimal hop count, which means that the exhaustive search range of the optimal hop count can be significantly reduced. Potential extensions of this paper include addressing a wider scenario with dissimilar sizes and densities of the SBS clusters.

\section{Competing Interests}

The authors declare that they have no competing interests. 


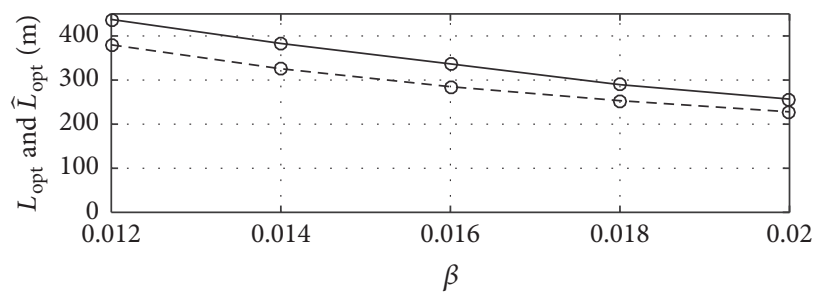

- Optimal $L_{\text {opt }}$

- $\ominus$ - Suboptimal $\widehat{L}_{\text {opt }}$

(a)

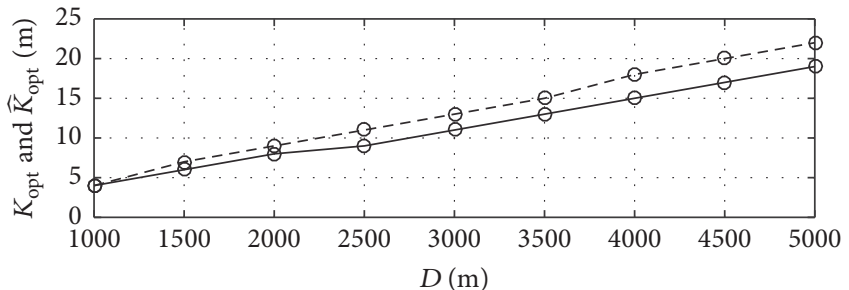

- Optimal $K_{\mathrm{opt}}$

- $\ominus$ - Suboptimal $\widehat{K}_{\text {opt }}$

FIGURE 7: Optimal hop distance and hop count for $\lambda=0.003$ and $b_{k}=4$ : (a) $L_{\text {opt }}$ versus $\beta$ and (b) $K_{\text {opt }}$ versus $D$, when $\beta=0.02$.

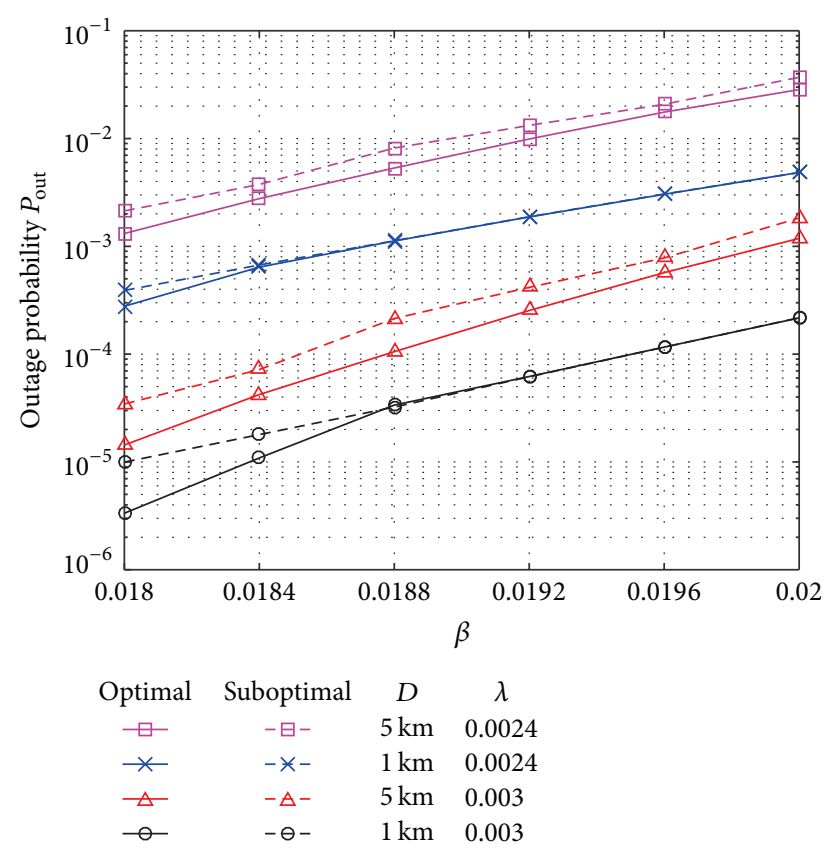

Figure 8: $P_{\text {out }}$ versus $\beta$, when $b_{k}=4, \lambda=\{0.0024,0.003\}$, and $D=$ $\{1 \mathrm{~km}, 2 \mathrm{~km}\}$.

\section{Acknowledgments}

This research was supported by Basic Science Research Program through the National Research Foundation of Korea (NRF) funded by the Ministry of Education (Grant no.: NRF2016R1D1A1B03930060).

\section{References}

[1] U. Siddique, H. Tabassum, E. Hossain, and D. I. Kim, "Wireless backhauling of $5 \mathrm{G}$ small cells: challenges and solution approaches," IEEE Wireless Communications, vol. 22, no. 5, pp. 22-31, 2015.

[2] W. Ni, I. B. Collings, X. Wang, and R. P. Liu, "Multi-hop pointto-point FDD wireless backhaul for mobile small cells," IEEE Wireless Communications, vol. 21, no. 4, pp. 88-96, 2014.

[3] X. Ge, H. Cheng, M. Guizani, and T. Han, "5G wireless backhaul networks: challenges and research advances," IEEE Network, vol. 28 , no. 6, pp. 6-11, 2014.
[4] Z. Pi and F. Khan, "An introduction to millimeter-wave mobile broadband systems," IEEE Communications Magazine, vol. 49, no. 6, pp. 101-107, 2011.

[5] S. Hur, T. Kim, D. J. Love, J. V. Krogmeier, T. A. Thomas, and A. Ghosh, "Millimeter wave beamforming for wireless backhaul and access in small cell networks," IEEE Transactions on Communications, vol. 61, no. 10, pp. 4391-4403, 2013.

[6] T. S. Rappaport, S. Sun, R. Mayzus et al., "Millimeter wave mobile communications for 5G cellular: it will work!" IEEE Access, vol. 1, pp. 335-349, 2013.

[7] T. S. Rappaport, F. Gutierrez, E. Ben-Dor, J. N. Murdock, Y. Qiao, and J. I. Tamir, "Broadband millimeter-wave propagation measurements and models using adaptive-beam antennas for outdoor Urban cellular communications," IEEE Transactions on Antennas and Propagation, vol. 61, no. 4, pp. 1850-1859, 2013.

[8] S. Rajagopal, S. Abu-Surra, and M. Malmirchegini, "Channel feasibility for outdoor non-line-of-sight mmwave mobile communication," in Proceedings of the IEEE Vehicular Technology Conference (VTC Fall '12), pp. 1-6, Quebec City, Canada, September 2012.

[9] J. G. Andrews, S. Buzzi, W. Choi et al., "What will 5G be?" IEEE Journal on Selected Areas in Communications, vol. 32, no. 6, pp. 1065-1082, 2014.

[10] S. Y. Seidel and T. S. Rappaport, "Site-specific propagation prediction for wireless in-building personal communication system design," IEEE Transactions on Vehicular Technology, vol. 43, no. 4, pp. 879-891, 1994.

[11] A. Toscano, F. Bilotti, and L. Vegni, "Fast ray-tracing technique for electromagnetic field prediction in mobile communications," IEEE Transactions on Magnetics, vol. 39, no. 3, pp. 12381241, 2003.

[12] T. Bai, R. Vaze, and R. W. Heath, "Analysis of blockage effects on urban cellular networks," IEEE Transactions on Wireless Communications, vol. 13, no. 9, pp. 5070-5083, 2014.

[13] A. Ghosh, N. Mangalvedhe, R. Ratasuk et al., "Heterogeneous cellular networks: from theory to practice," IEEE Communications Magazine, vol. 50, no. 6, pp. 54-64, 2012.

[14] T. Bai and R. W. Heath, "Coverage and rate analysis for millimeter-wave cellular networks," IEEE Transactions on Wireless Communications, vol. 14, no. 2, pp. 1100-1114, 2015.

[15] J. Choi, "On the macro diversity with multiple BSs to mitigate blockage in millimeter-wave communications," IEEE Communications Letters, vol. 18, no. 9, pp. 1623-1656, 2014.

[16] H. Jung and I. Lee, "Outage analysis of millimeter-wave wireless backhaul in the presence of blockage," IEEE Communications Letters, vol. 20, no. 11, pp. 2268-2271, 2016. 
[17] P. Billingsley, Probability and Measure, John Wiley \& Sons, 2012.

[18] J. G. Andrews, F. Baccelli, and R. K. Ganti, "A tractable approach to coverage and rate in cellular networks," IEEE Transactions on Communications, vol. 59, no. 11, pp. 3122-3134, 2011.

[19] I.-H. Lee and D. Kim, "Spatial channel reuse in multi-hop decode-and-forward relaying systems in high path loss environments," IEEE Communications Letters, vol. 16, no. 7, pp. 990 993, 2012. 


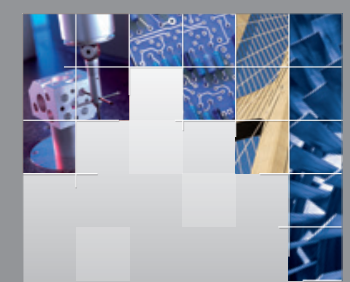

\section{Enfincering}
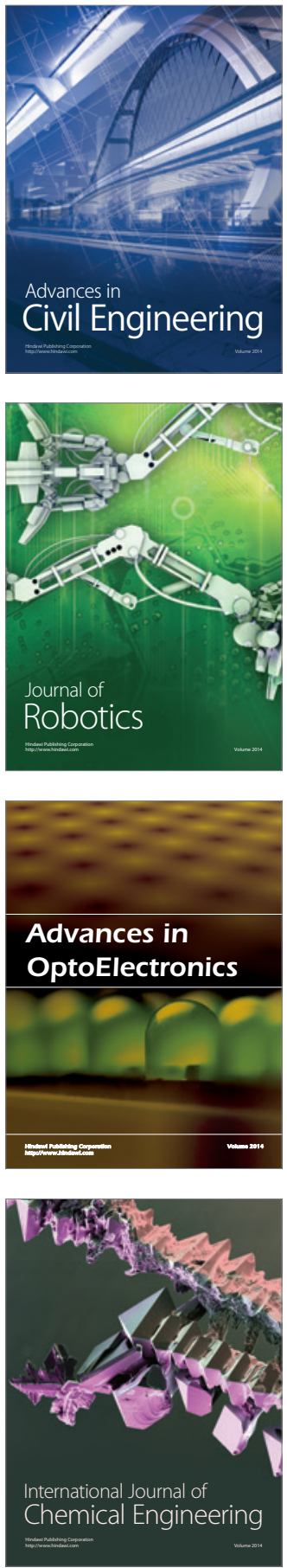

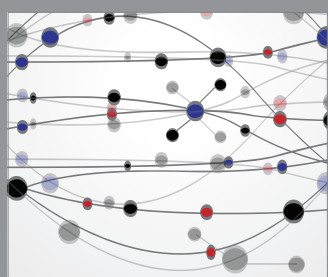

The Scientific World Journal

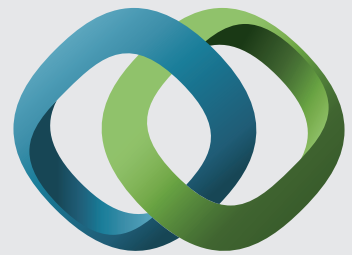

\section{Hindawi}

Submit your manuscripts at

https://www.hindawi.com
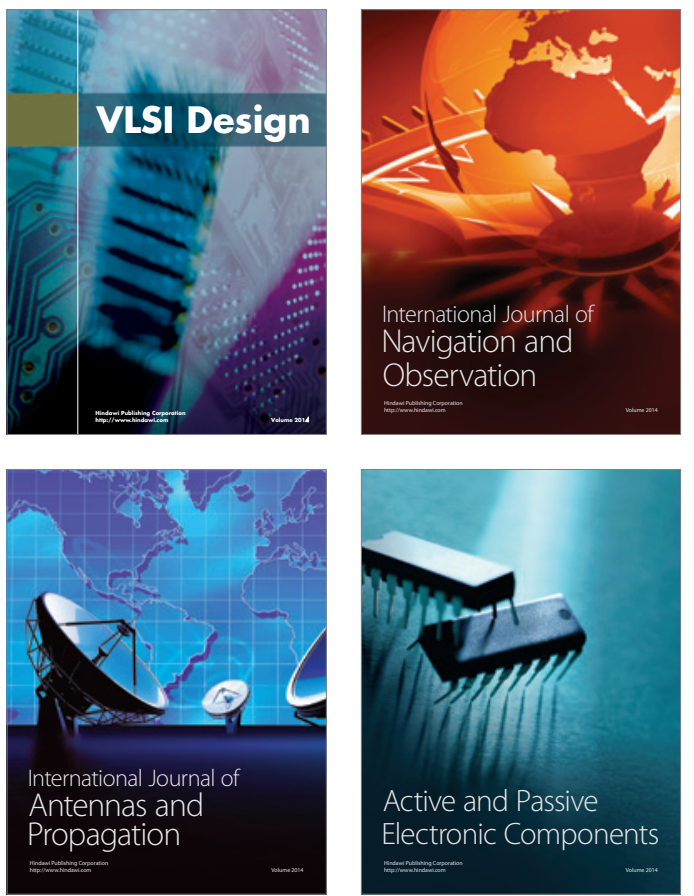
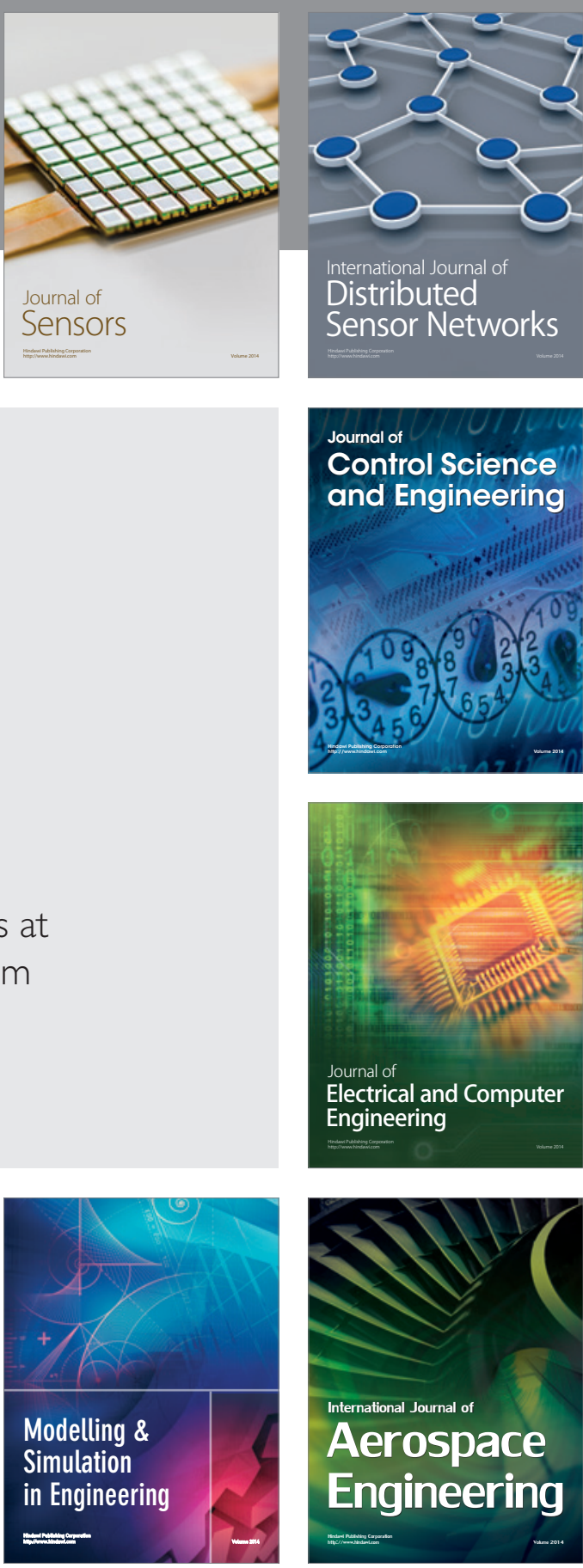

International Journal of

Distributed

Sensor Networks

$-$

Joumal of

Control Science

and Engineering
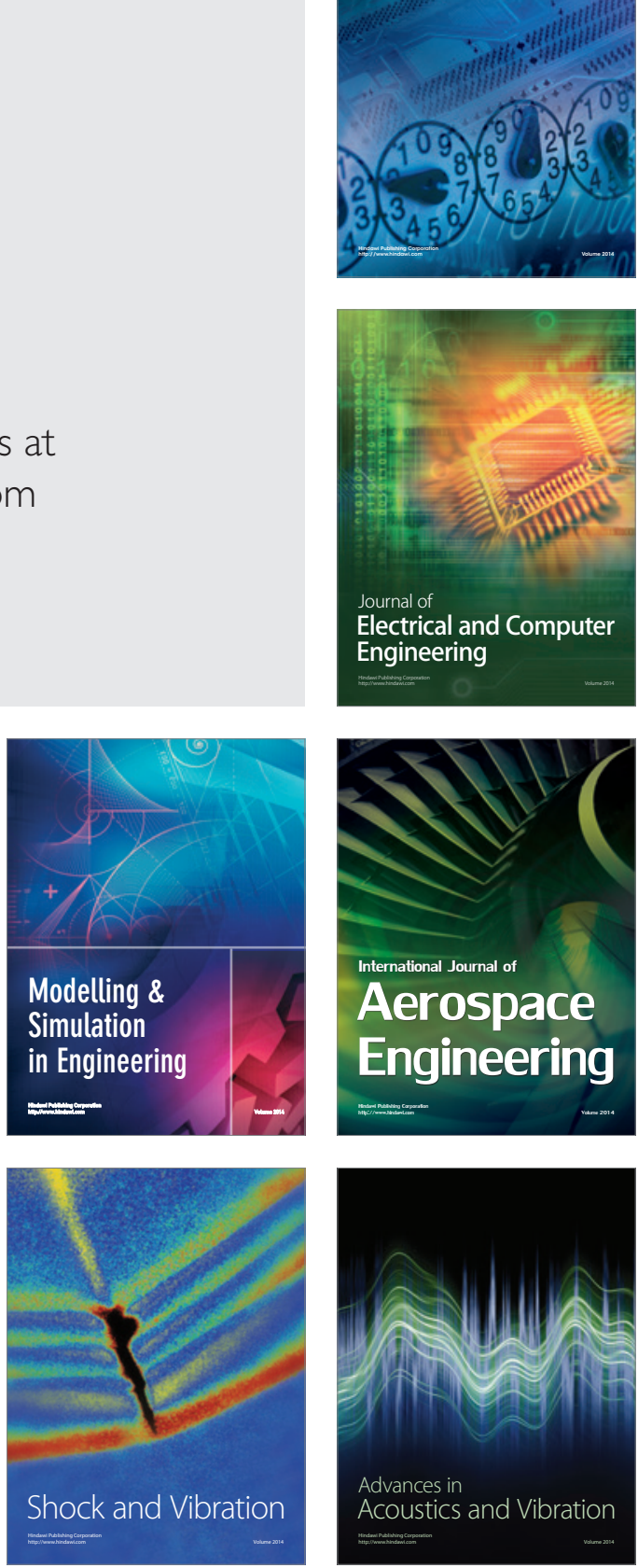